Notice de l'éditrice (Français)

Nota de la editora (Español)

Editor's Notes (English)

Nota de l'editora (Català)

\title{
Notice de l'éditrice (Français)
}

On peut se tourner dans l'histoire et vers le philosophe grec Aristote pour trouver les préoccupations philosophiques sur la signification du concept de théorie et de la relation entre la théorie, la connaissance et la pratique. Ce qui est clair cependant, c'est que, même aujourd'hui, les experts ne sont pas entièrement sûrs de savoir comment acquérir des connaissances et comment les enseignants relient leurs connaissances théoriques à la pratique, qui sont tous deux des éléments essentiels de l'enseignement (Oonk, 2009). L'importance de cette relation est évidente dans l'article de notre auteur invité. Basé sur une recherche ethnographique menée à l'Université Autonome de Barcelone, Bastidas Arteaga décrit et analyse les résultats de ses observations de deux classes de méthodes pour les enseignants en langues étrangères. L'auteur fait ensuite des suggestions sur la façon d'intégrer les approches inductive et déductive à la formation des enseignants de langues.

Le rôle de l'enseignant continue d'être le centre d'attention pour le prochain article, écrit par Navarro, mais dans ce cas, le rôle de l'enseignant est impliqué en tant qu'agent de la mise en œuvre du mouvement éducatif interdisciplinaire connu comme "l'écriture à travers le programme d'études". L'auteur analyse un programme d'écriture à partir de perspectives différentes, la dimension institutionnelle (organisation de l'école, les programmes, les horaires) et la dimension méthodologique (méthode d'enseignement, des séquences pédagogiques, des dynamiques de classe), en combinaison avec une analyse des discussions des enseignants, des observations en classe et des objets réalisés par les étudiants dans le programme.

Le troisième article met l'accent en l'apprentissage des langues en collaboration, et en particulier en le travail de lecture à haute voix entre des pairs. Grâce aux données recueillies d'enregistrements des interactions entre les étudiants et les entretiens avec les élèves, Tost démontre les avantages de ce type de collaboration dans le processus d'apprentissage des langues. Enfin, la lecture et l'écriture surgissent aussi dans l'article final. En utilisant une approche ethnographique qualitative, Pérez-Peitx explore la pensée de deux enseignants de maternelle sur ce qui concerne l'enseignement et l'apprentissage de la lecture et de l'écriture, au meme temps que la relation entre les croyances et les pratiques dans la salle de classe. Dans la section sur les entretiens, Olid Baez réalise une interview très intéressante avec l'écrivain, chercheur et professeur Gil Txabi Arnal, qui traite, entre autres, le sujet de la mort dans la 
littérature pour enfants. Nous concluons notre volume avec le critique, écrit par Cerarols Anguera, de livre Telecollaboration 2.0. Language, Literacies and Intercultural Learning in the 21st Century, édité par Guth et Helm.

La recherche en éducation est importante pour aider les enseignants à améliorer leur capacité de résolution de problèmes et ses compétences en leadership, tandis que contribue à la croissance professionnelle continue et à la promotion de l'objectivité et la pensée critique dans la salle de classe. Et, même si c'est assez évident, mais peut-être plus important encore, la recherche en éducation conduit à une amélioration de l'enseignement et l'apprentissage. Au sein de ces paramètres, les articles inclus dans le volume de 6,1 Bellaterra Journal of Teaching \& Learning Language \& Literature sont contributions à la compréhension croissante des processus complexes d'enseignement et d'apprentissage de la langue et de la littérature.

Certains de nos lecteurs auront remarqué que nous avons commencé en 2013 avec une nouvelle couverture. Nous sommes très heureux de cette nouvelle apparence et attrayant qui a été géré par des étudiants EINA conception et espérons que aimé nos lecteurs aussi!

Dra. Melinda Dooly

3 février 2013

\section{Crédits}

Les illustrations des couvertures de chaque volume ont été conçues par des étudiants de l'école EINA (Escola de Disseny i Art, Barcelona), dans le cadre du programme d'études supérieures 'Illustration créative', sous la direction de Sonia Pulido, professeure d'illustration de presse.

\section{Au comité de lecture du volume 6.1}

L'éditrice remercie les personnes suivantes pour leur contribution à la préparation du ce volume:

Llorenç Comajoan, Javier Elvira, Olga Esteve, Xavier Fontich, Ana Maria Margallo, Marta Milian, Luci Nussbaum et Virginia Unamuno.

\section{Références:}

Oonk, W. (2009). Theory-enriched practical knowledge in mathematics teacher education, PhD thesis, Leiden University Graduate School of Teaching, Leiden University, NL. 


\section{Nota de la editora (Español)}

Es posible remontarse en la historia hasta el filósofo griego Aristóteles para hallar reflexiones filosóficas sobre cuál es el significado del concepto teoría y la relación entre ella, el conocimiento y la práctica. A pesar de ello, lo que está claro es que incluso hoy en día, los expertos no están completamente seguros sobre cómo los profesores adquieren conocimientos y cómo vinculan sus conocimientos teóricos con la práctica, lo cual es fundamental para la enseñanza (Oonk, 2009). La importancia de esta relación es evidente en el artículo de nuestro autor invitado. Basándose en una investigación etnográfica realizada en la Universitat Autònoma de Barcelona, Bastidas Arteaga describe y analiza los resultados de sus observaciónes en dos clases de metodología para profesores de lenguas extranjeras para sus sugerencias sobre la manera de integrar los enfoques inductivos y deductivos en la educación de los profesores de lenguas.

El papel del maestro sigue siendo el foco de atención para el artículo siguiente, escrito por Navarro. En este caso, el papel del profesorado es implícito, como agentes del movimiento educativo interdisciplinar conocido como "escritura a través del currículo". El autor analiza un programa de escritura desde diferentes perspectivas: la dimensión institucional (organización escolar, programa de estudios, horarios) y la dimensión metodológica (método de enseñanza, secuencias didácticas, dinámica de clase), en combinación con el análisis de los debates de los profesores, observaciones de clase y artefactos producidos por los estudiantes dentro del programa.

El tercer artículo cambia de enfoque para mirar hacia la colaboración en el aprendizaje de idiomas y en particular, en el ejercicio de lectura en voz alta realizada entre pares. Utilizando datos obtenidos en las grabaciones de las interacciones entre los estudiantes y las entrevistas con ellos, Tost demuestra las ventajas de este tipo de colaboración en el proceso de aprendizaje de idiomas. Finalmente, tanto la lectura como la escritura surgen en el artículo final. Utilizando un enfoque cualitativo etnográfico, Pérez-Peitx explora el 'sistema de creencias' de dos maestros de educación infantil sobre la enseñanza y el aprendizaje de la lectura y la escritura así como la relación entre sus creencias y sus prácticas en el aula. En el apartado de entrevistas, Olid Báez lleva a cabo una interesante discusión con el escritor, investigador y profesor Txabi Gil Arnal, que trata, entre otras cosas, el tema de la muerte en la literatura infantil. Terminamos nuestro volumen con la reseña, escrita por Anguera Cerarols, del libro Telecollaboration 2.0. Language, Literacies and Intercultural Learning in the 21st Century, coordinado por Guth y Helm.

La investigación en educación es importante porque ayuda a los profesores a aumentar su capacidad para resolver problemas y sus habilidades de liderazgo. También contribuye al crecimiento profesional continuo y a la promoción de la objetividad y el pensamiento crítico en el aula. Y aunque bastante obvio quizás lo más importante, la investigación en educación 
conduce a la mejora de la enseñanza y el aprendizaje. Dentro de estos parámetros, los artículos incluidos en el volumen de 6,1 de Bellaterra Journal of Teaching \& Learning Language \& Literature son contribuciones a la comprensión cada vez mayor de los intrincados procesos de enseñanza y aprendizaje de la lengua y la literatura.

Algunos de nuestros lectores habrán notado que empezamos en el año 2013 con una portada nueva. Estamos muy entusiasmados con esta nueva y atractiva apariencia que ha sido ejecutado por los estudiantes de diseño de EINA y esperamos que les guste a nuestros lectores también.

Dra. Melinda Dooly

3 febrero de 2013

\section{Créditos}

Ilustraciones para las portadas de cada edición son diseñadas por los estudiantes de EINA (Escuela de Diseño y Arte, Barcelona), dentro del programa del posgrado Ilustración Creativa, bajo la dirección de Sonia Pulido, profesor de Ilustración de Prensa.

\section{Revisores del volumen 6.1}

La editora agradece a las siguientes personas por su contribución para la preparación de este volumen:

Llorenç Comajoan, Javier Elvira, Olga Esteve, Xavier Fontich, Ana Maria Margallo, Marta Milian, Luci Nussbaum y Virginia Unamuno.

\section{Referencias:}

Oonk, W. (2009). Theory-enriched practical knowledge in mathematics teacher education, $\mathrm{PhD}$ thesis, Leiden University Graduate School of Teaching, Leiden University, NL. 


\section{Editor's Notes (English)}

One can go all the way back to the Greek philosopher Aristotle to find philosophical concerns about what is the meaning of the concept of theory and the relationship between theory, knowledge and practice. What is clear, however, is that even today, scholars are uncertain about exactly how teachers construct knowledge and then link theoretical knowledge and practical situations, both of which are vital components of teaching (Oonk, 2009). The relevance of understanding this relationship emerges in our guest author's article. Based on ethnographic research carried out at the Universitat Autònoma de Barcelona, Bastidas Arteaga outlines and analyses the results of observation of two classes of methodology for foreign language teachers. The author then makes suggestions about how to integrate inductive and deductive approaches to language teacher education.

The role of the teacher continues to be the focus of attention for the next article, written by Navarro, however, in this case it is implicit -as agents for the implementation of the multidisciplinary educational movement known as "writing across the curriculum". The author analyses a writing program implemented in a teaching institution from different perspectives institutional dimension (school organization, study programme, timetable) and methodological (teaching approach, teaching sequences, class dynamics), combined with analysis of teacher discussions, class observations and student output.

The third article shifts the focus to peer collaboration in language learning, specifically collaborative work between partners reading out loud to each other. Using recordings of student interactions and student interviews, Tost demonstrates the advantages of this type of collaborative work in the language learning process. Both reading and writing come up again in the final article. Using a qualitative ethnographic approach, Pérez-Peitx explores two kindergarten teachers' 'systems of beliefs' about teaching and learning reading and writing, and how these related to their actual practice in the classroom. Next Olid Báez carries out an interesting interview with writer, researcher and teacher Txabi Arnal Gil, who discusses, among other things, the topic of 'death' in children's literature. We end our volume with Anguera Cerarols' book review of Telecollaboration 2.0. Language, Literacies and Intercultural Learning in the 21st Century, edited by Guth and Helm.

Research in education helps teachers gain problem-solving and leadership skills and enhances their continuous professional growth as well promoting objectivity and critical thinking in the classroom. And, although quite obvious, perhaps most importantly, research in education leads to improvement in teaching and learning situations. Along these lines, the articles in volume 6.1 of Bellaterra Journal of Teaching \& Learning Language \& Literature are contributions to the growing understanding of the intricate processes of teaching and learning language and literature. 
Some of our readers may have noticed that we start off the year 2013 with a revamped cover. We are excited about this bold new look which has been implemented by the talented design students from EINA and hope that our readers like it as well!

Dr. Melinda Dooly

3 February 2013

\section{Credits:}

Illustrations for the covers of each issue are designed by students of EINA (Escola de Disseny $i$ Art, Barcelona) studying in the postgraduate course 'Il-lustració Creativa', under the direction of Sonia Pulido, teacher of Illustration for Publishing Media.

\section{Reviewers for Volume 6.1}

The editor would like to thank the following reviewers for their contribution to the preparation of this volume:

Llorenç Comajoan, Javier Elvira, Olga Esteve, Xavier Fontich, Ana Maria Margallo, Marta Milian, Luci Nussbaum and Virginia Unamuno.

\section{Works Cited:}

Oonk, W. (2009). Theory-enriched practical knowledge in mathematics teacher education, $\mathrm{PhD}$ thesis, Leiden University Graduate School of Teaching, Leiden University, NL. 


\section{Nota de l'editora (Català)}

Es pot remuntar en la història fins al filòsof grec Aristòtil per trobar-hi reflexions filosòfiques sobre quin és el significat de teoria i la relació entre ella, el coneixement i la pràctica. El que està clar, però, és que fins i tot avui en dia, els experts no estan completament segurs sobre cóm els professors adquireixen coneixement i cóm vinculen els seus coneixements teòrics amb la pràctica, la qual cosa és fonamental per a l'ensenyament (Oonk, 2009). La importància d'aquesta relació és evident en l'article del nostre autor convidat. Basat en una investigació etnogràfica realitzada a la Universitat Autònoma de Barcelona, Bastidas Arteaga descriu i analitza els resultats de les seves observacions en dues classes de metodologia per a professors de llengües estrangeres, per acabar amb suggeriments sobre la manera d'integrar els enfocaments inductius i deductius a la formació de professors de llengües.

El paper del mestre segueix sent el focus d'atenció per al següent article, escrit per Navarro, però en aquest cas, el paper del professorat és implícit com a agents del moviment educatiu interdisciplinari conegut com a "escriptura a través del currículum". L'autor analitza un programa d'escriptura des de diferents perspectives com la dimensió institucional (organització escolar, programa d'estudis, horaris) i la dimensió metodològica (mètode d'ensenyament, seqüències didàctiques, la dinàmica de classe), en combinació amb l'anàlisi dels debats dels professors, observacions de classe i artefactes produïts pels estudiants dins del programa.

El tercer article canvia d'enfocament per mirar a la col-laboració en l'aprenentatge d'idiomes i en particular, a la col-laboració entre parells durant els exercicis de lectura en veu alta. Tot utilitzant dades recopilades en els enregistraments de les interaccions entre els estudiants i les entrevistes amb els estudiants, Tost demostra els avantatges d'aquest tipus de col-laboració en el procés d'aprenentatge d'idiomes. Finalment, tant la lectura com l'escriptura sorgeixen en el l'article final. Utilitzant un enfocament qualitatiu etnogràfic, Pérez-Peitx explora el 'sistema de creences' de dues mestres d'educació infantil sobre l'ensenyament i l'aprenentatge de la lectura i l'escriptura i la relació entre les seves creences i les seves pràctiques a l'aula. En l'apartat d'entrevistes, Olid Báez porta a terme una interessant discussió amb l'escriptor, investigador i professor Txabi Gil Arnal, que tracta, entre altres coses, el tema de la mort en la literatura infantil. Acabem el nostre volum amb la ressenya, escrita per Anguera Cerarols, del llibre Telecollaboration 2.0. Language, Literacies and Intercultural Learning in the 21st Century, coordinat per Guth i Helm.

La investigació en educació és important per ajudar als professors a augmentar la seva capacitat per resoldre problemes i les seves habilitats de lideratge. També contribueix al creixement professional continu i la promoció de l'objectivitat i el pensament crític a l'aula. I encara que és bastant obvi però potser el més important, la investigació en educació condueix a la millora de l'ensenyament i l'aprenentatge. Dins d'aquests paràmetres, els articles inclosos en 
el volum de 6,1 de Bellaterra Journal of Teaching \& Learning Language \& Literature són contribucions a la comprensió cada vegada més gran dels intricats processos d'ensenyament i aprenentatge de la llengua i la literatura.

Alguns dels nostres lectors hauran notat que vam començar l'any 2013 amb una portada nova. Estem molt entusiasmats amb aquest nova i atractiva aparença que ha estat executat pels estudiants de disseny d'EINA i esperem que els agrada als nostres lectors també.

Dra. Melinda Dooly

3 febrer de 2013

\section{Crèdits}

Les Il•lustracions per a les portades de cada edició són dissenyades pels estudiants d'EINA (Escola de Disseny i Art, Barcelona), dins del programa del postgrau Il-lustració Creativa, sota la direcció de Sonia Pulido, professora d'Il·lustració de Premsa.

\section{Els revisors de volum 6.1}

L'editora agraeix a les següents persones la seva contribució a la preparació d'aquest volum:

Llorenç Comajoan, Javier Elvira, Olga Esteve, Xavier Fontich, Ana Maria Margallo, Marta Milian, Luci Nussbaum and Virginia Unamuno.

\section{Referències:}

Oonk, W. (2009). Theory-enriched practical knowledge in mathematics teacher education, $\mathrm{PhD}$ thesis, Leiden University Graduate School of Teaching, Leiden University, NL. 